\title{
ПРАКТИЧЕСКИЕ РЕКОМЕНДАЦИИ ПО ЛЕЧЕНИЮ ПОГРАНИЧНЫХ ОПУХОЛЕЙ ЯИЧНИКОВ
}

Коллектив авторов: Давыдова И.Ю., Ашрафян Л.А., Карселадзе А.И., Кузнецов В. В., Максимов С.Я., Новикова Е.Г., Урманчеева А.Ф., Чекалова М.А.

DOI: $10.18027 / 2224-5057-2020-10-3 s 2-12$

Ключевые слова: пограничные опухоли яичников, атипически пролиферирующие опухоли, серозные пограничные опухоли, неинвазивная микропапиллярная низкой степени злокачественности (low grade) серозная карцинома, муцинозная пограничная опухоль, эдометриоидная пограничная опухоль

\section{1. ПОГРАНИЧНЫЕ ОПУХОЛИ ЯИЧНИКОВ (ОБЩАЯ ХАРАКТЕРИСТИКА)}

\section{1. Определение}

Пограничные опухоли яичников (ПОя) или атипически пролиферирующие опухоли - новообразования с атипичной пролиферацией эпителия без деструктивной стромальной инвазии (за некоторым исключением - см. ниже). поя составляют 15-20\% всех эпителиальных новообразований яичников. Наиболее распространенными гистотипами Поя являются серозные и муцинозные. Эндометриоидные опухоли, опухоли Бреннера, светлоклеточные, серо-муцинозные пограничные опухоли встречаются редко (3-4\%).

В отличие от рака яичников, пограничные опухоли, как правило, определяются на ранних стадиях заболевания и чаще обнаруживаются у женщин пременопаузального возраста. Больные поя в целом на 10 лет моложе пациенток, страдающих раком яичников (45 и 55 лет). Одним из факторов риска развития поя является бесплодие, в то время как беременность и лактация, напротив, обладают протективным действием. Установление диагноза Поя является прерогативой исключительно морфологического исследования и представляет непростую задачу. У трети больных ПОЯ моложе 40 лет возможно выполнение органосохраняющих операций. В большинстве исследований, посвященных изучению ПОЯ, сообщается о неэффективности ХТ. ПОЯ в большинстве наблюдений характеризуются благоприятным прогнозом.

\section{2. Серозные пограничные опухоли яичников}

Серозные пограничные опухоли яичников (СПОЯ) составляют 53-65\% всех ПоЯ, развиваются преимущественно у женщин репродуктивного возраста

Цитирование: Давыдова И.Ю., Ашрафян Л.А., Карселадзе А.И., Кузнецов В.В., Максимов С.Я., Новикова Е.Г. и соавт. Практические рекомендачии по лечению пограничных опухолей яичников. Злокачественные опухоли: Практические рекомендации RUSSCO \#3s2, 2020 (том 10).12 
и характеризуются, в большинстве своем, благоприятным прогнозом. Именно для серозного гистотипа Поя присущ ряд признаков, не характерных для остальных ПОЯ. К ним относятся билатеральность (40\%), перитонеальные импланты (30\%), возможность микроинвазии (инвазия опухоли протяженно-

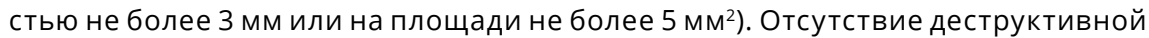
стромальной инвазии характеризует все гистотипы ПОЯ. Несмотря на то, что одним из симптомов споя является бесплодие, тем не менее, у пациенток с этой патологией может наблюдаться беременность. У беременных пациенток с СПОЯ микроинвазивный рост наблюдается в $80 \%$ случаев. Это следует учитывать при морфологической оценке опухоли, поскольку усугубление диагноза может привести к необоснованно завышенным объемам лечения и прерыванию желанной беременности. Стромальная микроинвазия СПоя не ухудшает прогноз, консервативные объемы хирургических вмешательств являются вполне приемлемыми у данной группы пациенток репродуктивного возраста. Вопрос о делении имплантов на инвазивные и неинвазивные в настоящее время трактуются более детально с привлечением большого количества сугубо морфологических признаков. Наличие большого количества инвазивных имплантов на сегодняшний день рассматривается как рак низкой степени злокачественности (low grade). У $27 \%$ больных клетки серозной пограничной опухоли можно обнаружить в лимфоузлах, однако, в отличие от злокачественных опухолей, на прогноз это не влияет. СПоя могут сопровождаться эндосальпингозом, доброкачественным процессом, характеризующимся дистопией эпителия трубного типа по серозной оболочке (брюшина, матка, яичники).

Микропапиллярный вариант СПОя (неинвазивная микропапиллярная серозная карцинома низкой степени злокачественности, low grade) составляет 6-26\% всех серозных пограничных опухолей. Микропапиллярные споя чаще встречаются при распространенных стадиях заболевания. При микропапиллярном варианте СПоя доля инвазивных имплантов увеличивается. Инвазивные и неинвазивные импланты при микропапиллярном варианте Споя встречаются с одинаковой частотой - у $40 \%$ больных; у $20 \%$ пациенток отмечаются смешанные инвазивные и неинвазивные импланты. При микропапиллярном варианте СПоя наблюдается бо́льшая частота рецидивов по сравнению с типичным вариантом СПОЯ и менее благоприятный прогноз.

Прогноз при Споя зависит от стадии заболевания: при начальных стадиях болезни рецидивы развиваются в среднем у $5 \%$ больных, при распространенных - у $25 \%$ больных. Пятилетняя безрецидивная выживаемость при I-ІІ стадиях составляет 98\%, а при III-IV стадиях - 82-90\%.

Широкое внедрение органосохраняющих операций позволяет сохранить менструальную функцию у 95-100\% больных, а способность к спонтанным беременностям - у 40-72\% пациенток. Негативное влияние беременности на прогрессирование заболевания не установлено, поэтому вопрос о предстоя- 
щей беременности можно решать спустя 3-6 мес. после органосохраняющего лечения.

Пограничные опухоли яичников несерозных гистотипов (муцинозные, эндометриоидные, Бреннера, светлоклеточные), как правило, представляют собой односторонние образования, для которых не характерны перитонеальные импланты.

Таким образом, пограничные опухоли протекают относительно благоприятно, так как генетические изменения приводят к пролиферативным процессам, но не к метастазам.

\section{3. Мүцинозные пограничные опухоли яичников}

Муцинозные пограничные опухоли яичников (МПОя) составляют 35-45\% всех пограничных опухолей яичника и занимают второе место после серозных опухолей. Дифференциальная диагностика МПОЯ с инвазивным муцинозным раком яичника и метастазами в яичнике крайне затруднительна. Нередко метастазы злокачественной опухоли аппендикса в яичнике принимают за муцинозную пограничную опухоль яичника, в связи с чем аппендэктомия ранее являлась этапом хирургического лечения МПОЯ. В настоящее время аппендэктомия рекомендована только тем больным, у которых при внимательном осмотре аппендикса определяется патология. Обычно МПОЯ представлены односторонними опухолями крупных размеров. Тем не менее, встречаются и двусторонние опухоли (5\% случаев - при интестинальном типе МПОЯ и 30$40 \%$ - при эндоцервикальном типе согласно предыдущей гистологической классификации). Импланты для МПОЯ не характерны. Практически всегда МПОЯ диагностируются в І стадии. В случаях обнаружения имплантов при муцинозных опухолях в первую очередь необходимо исключать злокачественную природу первичной опухоли яичника или метастатическое его поражение. Одним из характерных осложнений МПоя является псевдомиксома брюшины. 10-летняя выживаемость при МПОЯ составляет $95 \%$.

\section{4. Кодирование по МКБ-10}

Злокачественное новообразование яичников (C 56)

\section{5. Классификация}

Международная гистологическая классификация (ВО3, 4-е издание, 2014 г.)

\section{Серозные опухоли:}

8442 / 1 Серозная пограничная / атипически пролиферирующая опухоль

8460 / 2 Серозная пограничная опухоль - микропапиллярный вариант / неинвазивная low grade серозная карцинома 


\section{Муцинозные опухоли:}

8472 / 1 Муцинозная пограничная / атипически пролиферирующая муцинозная опухоль

\section{Эндометриоидные опухоли:}

8380 / 1 Эндометриоидная пограничная / атипически пролиферирующая эндометриоидная опухоль

\section{Светлоклеточные опухоли:}

8313 / 1 Светлоклеточная пограничная / атипически пролиферирующая светлоклеточная опухоль

\section{Опухоли Бреннера:}

9000 / 0 Пограничная опухоль / атипически пролиферирующая опухоль Бреннера

\section{Серозно-муцинозные опухоли:}

8474 / 1 Серозно-муцинозная / атипически пролиферирующая серозно-муцинозная опухоль

\section{6. Стадирование}

Стадирование поя производится по классификации TNM (8-ое издание, 2017 г.) и FIGO (2009 г.), (табл. 1).

Таблица 1. Стадирование рака яичников по TNM и FIGO (8-ое издание, 2017 г.)

\begin{tabular}{|c|c|c|}
\hline TNM & FIGO & \\
\hline T1 & I & Опухоль ограничена яичниками \\
\hline T1a & IA & $\begin{array}{l}\text { Опухоль ограничена одним яичником, капсула не повреждена, нет опухолевых разрас- } \\
\text { таний на поверхности яичника, нет злокачественных клеток в асцитической жидкости } \\
\text { или смывах из брюшной полости }\end{array}$ \\
\hline $\mathrm{T} 1 \mathrm{~b}$ & IB & $\begin{array}{l}\text { Опухоль ограничена двумя яичниками, их капсулы не повреждены, нет опухолевых } \\
\text { разрастаний на поверхности яичников, нет злокачественных клеток в асцитической } \\
\text { жидкости или смывах из брюшной полости }\end{array}$ \\
\hline T1c & IC & $\begin{array}{l}\text { Опухоль ограничена одним или двумя яичниками и сопровождается любым из следую- } \\
\text { щих факторов }\end{array}$ \\
\hline T1c1 & IC1 & Повреждение капсулы во время операции \\
\hline T1c2 & IC2 & $\begin{array}{l}\text { Повреждение капсулы до операции или опухоль на поверхности яичника/маточной } \\
\text { трубы }\end{array}$ \\
\hline T1c3 & IC3 & Злокачественные клетки в асцитической жидкости или смывах с брюшины \\
\hline $\mathrm{T} 2$ & II & Опухоль поражает один или два яичника с распространением на малый таз \\
\hline T2a & $\| \mathrm{A}$ & Врастание и/или метастазирование в матку и/или в одну или обе маточные трубы \\
\hline $\mathrm{T} 2 \mathrm{~b}$ & IIB & Распространение на другие ткани таза \\
\hline
\end{tabular}




\begin{tabular}{|c|c|c|}
\hline TNM & FIGO & \\
\hline $\begin{array}{l}\text { T3 } \\
\text { и/или } \\
\text { N1 }\end{array}$ & III & $\begin{array}{l}\text { Опухоль поражает один или оба яичника с гистологически подтвержденными вну- } \\
\text { трибрюшинными метастазами за пределами таза и/или метастазами в регионарных } \\
\text { лимфатических узлах (внутренних, наружных и общих подвздошных, запирательных, } \\
\text { крестцовых или поясничных лимфоузлах) }\end{array}$ \\
\hline N1 & $\begin{array}{l}\text { IIIA1 } \\
\text { IIIA1 (i) } \\
\text { IIIA (ii) }\end{array}$ & $\begin{array}{l}\text { Метастазы только в забрюшинных лимфатических узлах } \\
\text { Метастазы в лимфатических узлах размерами до } 10 \text { мм } \\
\text { Метастазы в лимфатических узлах размерами более } 10 \text { мм }\end{array}$ \\
\hline T3а & IIIA2 & $\begin{array}{l}\text { Микроскопические, гистологически подтвержденные внутрибрюшинные метастазы за } \\
\text { пределами таза } \pm \text { метастазы в забрюшинных лимфатических узлах }\end{array}$ \\
\hline T3b & IIIB & $\begin{array}{l}\text { Макроскопические внутрибрюшинные метастазы за пределами таза до } 2 \text { см включи- } \\
\text { тельно в наибольшем измерении } \pm \text { метастазы в забрюшинных лимфатических узлах }\end{array}$ \\
\hline T3c & IIIC & $\begin{array}{l}\text { Внутрибрюшинные метастазы за пределами таза более } 2 \text { см в наибольшем измерении } \\
\pm \text { метастазы в забрюшинных лимфатических узлах (включая распространение опухоли } \\
\text { на капсулу печени и селезенки без поражения паренхимы органов) }\end{array}$ \\
\hline M1 & $\begin{array}{l}\text { IV } \\
\text { IVA } \\
\text { IVB }\end{array}$ & $\begin{array}{l}\text { Отдаленные метастазы (исключая внутрибрюшинные метастазы) } \\
\text { Плевральный выпот со злокачественными клетками } \\
\text { Метастазы в паренхиматозных органах и других органах вне брюшной полости (в том } \\
\text { числе паховых лимфатических узлах и лимфатических узлах за пределами брюшной } \\
\text { полости) }\end{array}$ \\
\hline
\end{tabular}

\section{2. КЛИНИЧЕСКАЯ КАРТИНА}

Поя не имеют специфической клинической картины. Больные могут предъявлять жалобы на увеличение живота, боли различной интенсивности, ациклические кровянистые выделения. Иногда опухоли могут проявляться бессимтомными образованиями в малом тазу, самостоятельно обнаруживаемыми пациентами. Сравнительно часто пограничные опухоли обнаруживаются при хирургических вмешательствах, связанных с иными причинами.

\section{3. ДИАГНОСТИКА}

Специфических серологических критериев для ПОЯ не существует. Тем не менее, уровень СА-125 у больных ПОЯ превышает норму в два раза.

УЗИ является чувствительным методом диагностики ПОЯ и с высокой долей вероятности позволяет заподозрить эпителиальную опухоль яичников. Метод является высокоинформативным, позволяющим выявить опухолевые изменения в яичниках на ранних этапах. Несмотря на схожесть изображений ПОЯ с серозным раком яичников, КТ и МРТ играют важную роль в дифференциальной диагностике.

Диагностика включает:

- сбор анамнеза, изучение клинических симптомов (боли внизу живота, увеличение живота, пальпируемая опухоль в брюшной полости, нарушение менструального цикла, бесплодие); 
- физикальное, в том числе гинекологическое обследование;

- биохимический и общеклинический анализы крови, общий анализ мочи; - определение уровня опухолевых маркеров СА-125, HЕ-4, РЭА, СА-19.9;

- рентгенологическое исследование органов грудной клетки;

- УзИ (абдоминальное и трансвагинальное);

- MPT/КТ брюшной полости, малого таза (по показаниям, не являются рутинными методами диагностики поя);

- обследование ЖКТ (ЭГДС, колоноскопия или ирригоскопия).

\section{4. ЛЕЧЕНИЕ ПОГРАНИЧНЫХ ОПУХОЛЕЙ ЯИЧНИКОВ}

Всем больным Поя показано хирургическое лечение. Химиотерапия не показана ни при одном гистотипе ПОЯ. Для успешного и эффективного консервативного лечения пограничных опухолей необходима высокая квалификация морфолога.

\section{1. Серозные пограничные опухоли яичников}

\subsection{1. Пациентки репродуктивного периода}

\section{IA-IC стадии, в том числе микропапиллярный вариант}

Пациенткам репродуктивного периода, желающим сохранить фертильность и гормональную функцию яичника, рекомендованы органосохраняющие операции в объеме резекции / аднексэктомии опухолево измененного яичника / яичников. При резекции яичника целесообразно интраоперационное гистологическое исследование (для исключения инвазивного рака низкой степени злокачественности, low grade).

Биопсия / клиновидная резекция контралатерального яичника при отсутствии в нем видимой патологии не показана. При отсутствии здоровой ткани в яичнике / яичниках показана одно / двусторонняя аднексэктомия, экстирпация матки с придатками.

Хирургический доступ: лапаротомный, лапароскопический.

Больным, не желающим сохранить репродуктивную и гормональную функцию яичника, рекомендована экстирпация матки с придатками. Резекция большого сальника, биопсия брюшины, взятие смывов брюшной полости выполняется всем больным.

\section{IIA-IIIB стадии}

Больным, желающим сохранить репродуктивную и гормональную функцию яичника, возможно выполнение органосохраняющих операций в объеме резекции яичника / яичников, аднексэктомии, при невозможности сохранения ткани яичников - одно / двусторонняя аднексэктомия, экстирпация матки 
с придатками. Больным, не желающим сохранить репродуктивную и гормональную функцию яичника, рекомендована экстирпация матки с придатками.

Резекция большого сальника, биопсия брюшины, взятие смывов брюшной полости выполняются всем больным.

Доступ: лапаротомный, лапароскопический

\section{IIIB-IIIC стадии (за исключением случаев выраженной диссеминации опухоли по париетальной и висцеральной брюшине брюшной полости)}

Больным, желающим сохранить репродуктивную и гормональную функцию яичника, возможно выполнение органосохраняющих операций в объеме резекции / аднексэктомии опухолево измененного яичника / яичников, удаления видимых узлов с брюшины.

При резекции яичника целесообразно интраоперационное гистологическое исследование яичника.

При невозможности сохранения здоровой ткани яичника - экстирпация матки с придатками.

Больным, не желающим сохранить репродуктивную и гормональную функцию яичника, рекомендована экстирпация матки с придатками.

Резекция большого сальника, биопсия брюшины, взятие смывов брюшной полости выполняется всем больным.

Доступ: преимущественно лапаротомный.

После выполнения органосохраняющего хирургического вмешательства можно рекомендовать беременность через 3-6 мес. (в зависимости от доступа) после операции.

\subsection{2. Пациентки, достигшие менопаузы}

Увеличение объема хирургического лечения не влияет на прогноз заболевания.

\section{IA-IIIA стадии}

Стандартным объемом хирургического лечения является экстирпация матки с придатками.

При выполнении аднексэктомии и выявлении пограничной опухоли при плановом гистологическом исследовании повторную операцию с целью рестадирования можно не выполнять при условии отсутствия подозрений на опухоль контралатерального яичника, отсутствия остаточной опухоли в брюшной полости.

При вовлечении в процесс яичников - двусторонняя аднексэктомия или экстирпация матки с придатками.

Резекция большого сальника, биопсия брюшины, взятие смывов брюшной полости показана всем больным.

Доступ: лапаротомный, лапароскопический. 


\section{IIIB-IIIC стадии}

Экстирпация матки с придатками, удаление видимых узлов с брюшины.

Резекция большого сальника, биопсия брюшины, взятие смывов брюшной полости показаны всем больным.

В том случае, если первичное хирургическое лечение проведено без удаления неизмененного большого сальника, рекомендовано наблюдение (УЗИ брюшной полости, малого таза, СА-125, НЕ-4).

При отсутствии по данным УЗИ / КТ / МРТ) признаков опухоли в большом сальнике, брюшной полости, яичнике / яичниках рекомендовано динамическое наблюдение.

При наличии макроскопических опухолевых узлов по брюшине, в большом сальнике показано удаление большого сальника, всех видимых узлов брюшной полости.

При неоптимальной (с остаточной опухолью) операции и выявлении после планового гистологического исследования инвазивного рака низкой степени злокачественности low grade, лечение проводится в соответствии с программой лечения рака яичников. Тактика лечения определяется на консилиуме с участием морфологов, онкогинекологов в экспертной клинике.

\section{2. Микропапиллярный вариант серозной ПОЯ}

Если операция изначально выполнялась без резекции большого сальника, то при микропапиллярном варианте Споя, независимо от данных обследования, в связи с высокой вероятностью инвазивных имплантов целесообразна релапаротомия / релапароскопия, удаление большого сальника, биопсия брюшины. Если при микропапиллярном варианте СПоя наблюдается диссеминация, рекомендована лапаротомия, экстирпация матки с придатками, удаление большого сальника, удаление диссеминатов брюшной полости (перитонэктомия).

\section{3. Лечение рецидивов СПОЯ}

\subsection{1. Рецидив в яичнике/ яичниках}

Рецидив в яичнике / яичниках возникает в 35-50\% наблюдений. У больных репродуктивного возраста (при желании сохранить фертильность) возможна ререзекция яичника / яичников, при отсутствии здоровой ткани яичника аднексэктомия, экстирпация матки с придатками. У больных менопаузального периода рекомендуется экстирпация матки с придатками. Резекция большого сальника, биопсия брюшины, взятие смывов брюшной полости выполняется всем больным, которым первоначально данная процедура не выполнялась.

Доступ: лапаротомный, лапароскопический. 


\subsection{2. Рецидив экстрагонадный/ экстрагенитальный}

Рецидив за пределами яичника / яичников (экстрагонадный, экстрагенитальный) встречается в 8-15\% наблюдений и может быть локальным, мультифокальным. Показано хирургическое лечение в объеме удаления рецидивных опухолевых узлов. У больных с изолированным экстрагонадным рецидивом (без опухолевого поражения яичника / яичников) после органосохраняющего хирургического лечения на первом этапе (резекция яичника / яичников, аднексэктомия) экстирпация матки с придатками не показана.

Повторные рецидивы требуют повторных хирургических вмешательств в объеме оптимальной циторедукции.

\section{4. Инвазивный рак низкой степени злокачественности (low grade) на фоне СПОЯ (ранние стадии)}

Молодым пациенткам, желающим сохранить репродуктивную и гормональную функцию, при клинической начальной стадии заболевания и поражении одного яичника возможно выполнение односторонней аднексэктомии. Рекомендовано выполнять процедуры стадирования (удаление большого сальника, биопсия брюшины, тазовая и поясничная лимфодиссекция, взятие смывов с брюшной полости для цитологического исследования) для исключения распространенной стадии заболевания.

Больным с начальными стадиями инвазивного рака низкой степени злокачественности (low grade), не желающим сохранить репродуктивную и гормональную функцию, рекомендовано выполнять экстирпацию матки с придатками, удаление большого сальника, процедуры стадирования.

Пациенткам с IA и IB стадией рака яичников степени злокачественности (low grade) адъювантное лекарственное лечение не показано.

Для больных с более распространенными стадиями лечение аналогично таковому для рака яичников (см. соответствующий раздел рекомендаций).

\section{5. Инвазивный рак низкой степени злокачественности (low grade) в диссеминатах}

Показана оптимальная циторедуктивная операция с дальнейшим лекарственным лечением в соответствии с рекомендациями по лечению рака яичников.

\section{6. Пограничные опухоли яичников несерозных гистотипов}

При одностороннем поражении рекомендована односторонняя аднексэктомия. При двустороннем процессе (редко - при МПОя) - двусторонняя аднексэктомия / экстирпация матки с придатками. Рекомендована также резекция большого сальника, биопсия брюшины, взятие смывов брюшной полости 
(сложность морфологической диагностики не всегда позволяет обнаружить фокусы аденокарциномы в пограничной опухоли).

В том случае, если первичное хирургическое лечение проведено без удаления неизмененного большого сальника, рекомендовано наблюдение (УзИ брюшной полости, малого таза, СА-125, НЕ-4, РЭА, СА-19.9).

При отсутствии признаков опухоли (по данным УЗИ или КТ или МРТ) в большом сальнике, брюшной полости, яичнике / яичниках рекомендовано динамическое наблюдение.

\section{5. ДИСПАНСЕРНОЕ НАБЛЮДЕНИЕ}

Тактика дальнейшего наблюдения зависит от исходной клинической ситуации и объема хирургического вмешательства.

\section{1. При серозном гистотипе ПОЯ}

\subsection{1. Больные, которым выполнены органосохраняющие операции}

- Наблюдение гинеколога, сбор анамнеза и жалоб, СА-125, УзИ брюшной полости и органов малого таза - 1 раз в 3-4 мес. в течение первых 5 лет, далее - 1 раз в 6-8 мес. В последующие 5 лет и далее - 1 раз в 12 мес. в течение 15 лет.

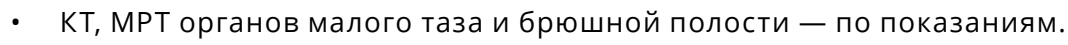

\subsection{2. Больные, которым выполнены пангистерэктомия, надвлагалищная ампутация матки с придатками, двусторонняя аднексэктомия}

- Наблюдение гинеколога, сбор анамнеза и жалоб, СА-125, УзИ брюшной полости и органов малого таза - 1 раз в 6 мес. в течение первых 5 лет, далее - 1 раз в 8-12 мес. В последующие 5 лет и далее - 1 раз в 12 мес. в течение 15 лет.

- $\mathrm{KT}, \mathrm{MPT}$ органов малого таза и брюшной полости - по показаниям.

\subsection{3. Больные после хирургического лечения диссеминированной серозной пограничной опухоли яичника}

- Наблюдение гинеколога, сбор анамнеза и жалоб, СА-125, УзИ брюшной полости и органов малого таза - 1 раз в 3-4 мес. в течение первых 5 лет, далее - 1 раз в 6-8 мес.; в последующие 5 лет и далее - 1 раз в 12 мес. в течение 15 лет.

- КТ, МРТ органов малого таза и брюшной полости - по показаниям 


\section{2. При несерозном гистотипе ПОЯ}

- Наблюдение гинеколога, сбор анамнеза и жалоб 1 раз 6 мес. первые 5 лет, далее - ежегодный профосмотр.

- УзИ органов малого таза - каждые 6 мес. в течение первых 5 лет.

\section{3. Возможность репродукции у больных ПОЯ}

Необходимо помнить, что молодым пациенткам, перенесшим органосохраняющее лечение по поводу поя, дальнейшая беременность и роды не противопоказаны. Рекомендовать беременность можно через 3-6 мес. после хирургического лечения (срок зависит от хирургического доступа). Абсолютных противопоказаний к применению вспомогательных репродуктивных технологий, назначению гормональных препаратов, комбинированных оральных контрацептивов нет. 